\title{
Simultaneous occurence of compound odontoma and arrested root formation as developmental disturbances after maxillofacial trauma: A case report
}

\author{
Metin Güngörmüş ${ }^{1}$, Ümit Yolcu ${ }^{2}$, Mutan-Hamdi Aras ${ }^{3}$, Koray Halicioğlu ${ }^{4}$ \\ ${ }_{1}^{1}$ Associate Professor, DDs, PhD, Department of Oral and Maxillofacial Surgery, Ataturk University. Erzurum/Turkey
${ }_{2}^{2}$ Research Assistant, DDs, Department of Oral and Maxillofacial Surgery, Ataturk University. Erzurum/Turkey
${ }^{3}$ Assistant Professor, DDs, PhD, Department of Oral and Maxillofacial Surgery, Gaziantep University. Gaziantep/Turkey
${ }^{4}$ Researcher, DDs, PhD, Department of Orthodontic, Ataturk University. Erzurum/Turkey
}

Correspondence:

Department of Oral and Maxillofacial Surgery

Faculty of Dentistry, Gaziantep University

Gaziantep, 27310

Turkey

mutanhamdi@hotmail.com

\author{
Güngörmüş M, Yolcu Ü, Aras MH, Halicioğlu K. Simultaneous occurence \\ of compound odontoma and arrested root formation as developmental dis- \\ turbances after maxillofacial trauma: A case report. Med Oral Patol Oral \\ Cir Bucal. 2010 Mar 1;15 (2):e398-400. \\ http://www.medicinaoral.com/medoralfree01/v15i2/medoralv15i2p398.pdf

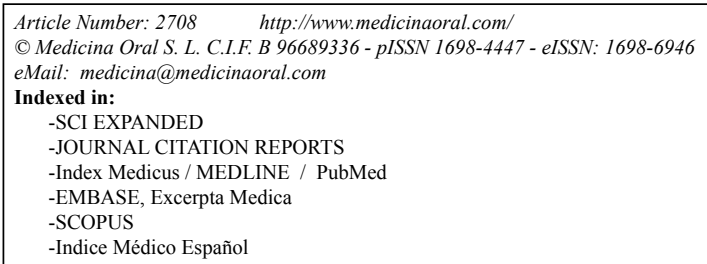

Received: $16 / 02 / 2009$

Accepted: 02/08/2009

\begin{abstract}
Traumatic injury to a primary tooth and/or a bone fracture has the potential to damage the underlying permanent tooth germ which may disturb its development. The extent of the malformation depends on the developmental stage of the permanent tooth and the intensity of the trauma. The presence of infection may be a predictive factor for these abnormalities. Open surgical procedures can also potentially cause impaction and developmental disturbances. Several developmental alterations such as discolouration, hypoplasia, crown dilaceration, root angulation or dilaceration, sequestration of permanent tooth buds and disturbance in eruption have been reported in permanent teeth after trauma. However, odontoma-like malformations and partial or complete arrest of root formation are rare complications developed after trauma. This article presents a rare case with simultaneous occurrence of an odontoma-like malformation and complete and partial arrested root formations as the results of maxillofacial trauma. Almost all pediatric fractures must be managed with closed reduction as much as possible. However, if it is necessary to perform an open reduction, careful attention must be paid during placement of the osteosynthetic plates and screws; and tooth bud development must be followed periodically.
\end{abstract}

Key words: Tooth bud, developmental disturbance, trauma, odontoma-like malformation, arrest root formation odontoma. 


\section{Introduction}

Bone fractures located in areas containing developing tooth germs can influence developing teeth's growth and maturation. Several developmental alterations, including white or yellow-brown discoloration, enamel hypoplasia, crown dilacerations, root duplication, vestibular root angulations, root angulations or dilacerations, sequestration of permanent tooth germs, disturbance in eruption, odontoma-like malformations and partial or complete arrest of root formation, have been reported in permanent teeth after dental and maxillofacial traumas. However, odontoma-like malformations and partial or complete arrest of root formation are rare complications developed after trauma (1-3).

The incidence of abnormalities in developing teeth involved in fractures were $18-45 \%$ in previous reports $(4,5)$. Although this incidence of abnormalities is somewhat high, most of the abnormalities did not significantly deleteriously affect dentition (2). Functional and esthetic disturbances can be caused by impaction; however, the incidence of impaction in previous studies was low, ranging from $0-16 \%(4)$. Thus, preserving the tooth buds involved in fractures is recommended. The presence of infection, however, is a predictive factor of abnormality. Open surgical procedures can be potential causes of impaction. The formation of odontoma and cysts has been reported previously (6).

The purpose of this paper is to report a case with simultaneous odontoma-like malformation and complete and partial arrested root formations, probably as the results of a trauma sustained in a traffic accident.

\section{Case Report}

A 14-year-old boy was referred to our department with a complaint of pain in his anterior mandibular area. Intraoral examination revealed that the anterior mandibular area was hyperemic and sensitive to palpation. The symptoms' etiology was possibly irritations of the miniplate. The left mandibular lateral incisor, canine and first premolar were not detected in the oral cavity. Radiological examination showed the unerupted left mandibular canine and first premolar and a miniplate associated with the unerupted left mandibular first premolar. There were complete or partial arrested root formations in these teeth and the right second molar. On the left side of the mandible, the second molar was formed normally; and there was a permanent third molar bud. On the right side there was no third molar bud, and an odontoma-like malformation was observed in this area. In addition, the right second molar's mesial root was completely formed, but showed deficient distal root formation; however, this tooth's response to an electric pulp test was within normal limits. Although a fracture line was present in the anterior segment, the trauma should have affected the right molar region indi- rectly. However, the deficient root formation and impaction of the left premolar could have resulted from open surgery. The mandibular first premolar's formation had progressed, but showed deficient root formation and impaction (Fig. 1). The left mandibular canine was embedded and its root's formation was partially arrested (Fig. 1). The patient gave a history of trauma (traffic accident) at age eight, for which he had been treated surgically at another hospital.

Under sedation and local anesthesia, the miniplate and screws in the anterior area, and the left mandibular canine and odontoma-like mass in the right ramus mandible, were removed (Fig. 2 and 3). Intraoperatively,

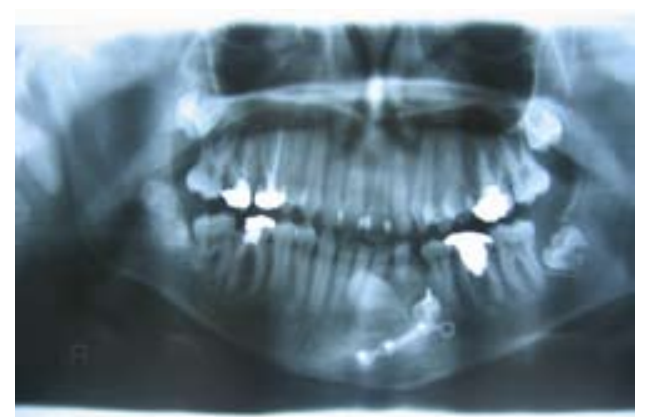

Fig. 1. Panoramic radiography of the patient.

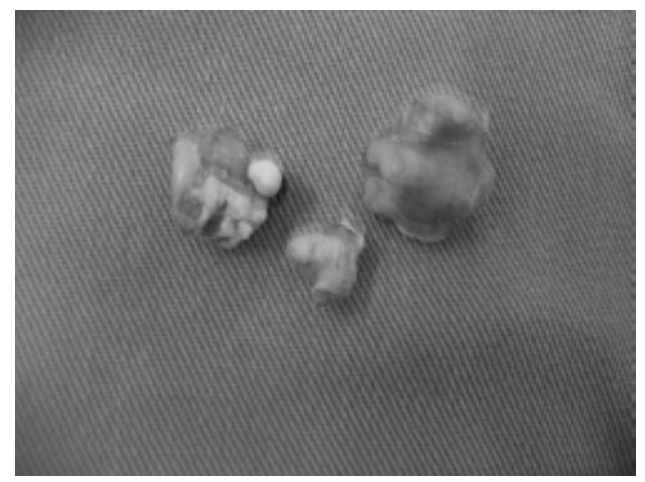

Fig. 2. Appearance of the odontoma-like malformation after its removal.

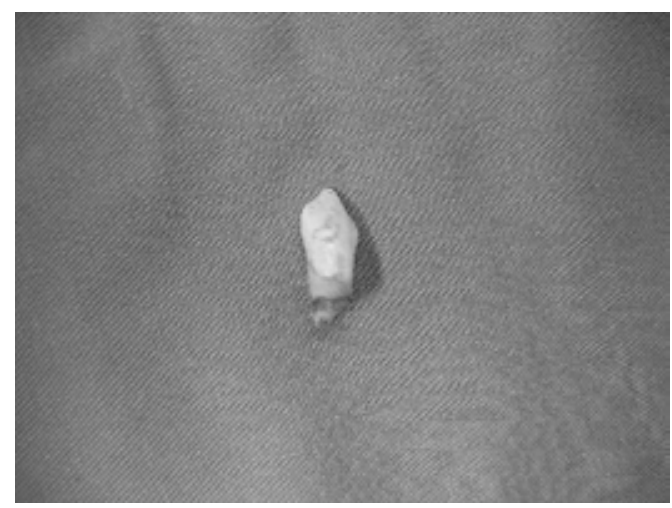

Fig. 3. Appearance of the mandibular canine teeth after their removal. 
we observed that one screw had been inserted under the mandibular first premolar's cervical line. Because any pathologic symptom related to this tooth was not evident radiologically, traction orthodontic of the first premolar was planned; and the patient was referred an orthodontist.

Histopathologic examination of the excised mass confirmed the diagnosis of compound odontoma.

\section{Discussion}

Any trauma during the stage of odontogenesis can seriously affect the morphogenetic stages of dental development, and several developmental alterations can arise in permanent teeth as a consequence of dental injuries and maxillofacial traumas. Odontoma-like malformations and partial or complete arrest of root formation are rare complications developed after trauma. The extent of these malformations depends on the permanent tooth's developmental stage. Odontoma-like malformations occur during early phases of odontogenesis (1). However, after a tooth's crown has formed, its root begins to develop; and malformations such as partial or complete arrest of root formation occur during root formation (2,3). Root formation of the cuspids, bicuspids and second permanent molar begins generally between 5-7 years of age; and hard tissue formation of the permanent mandibular third molar begins almost at the same time as these teeth. In our case, the trauma occurred at age eight, affecting root formation and the germs of the permanent successor, and leading to the development of an odontoma-like malformation and complete and partial arrested root formations. The formation of the left mandibular first premolar and canine had progressed with deficient root formation, and these teeth were embedded. Although a possible cause of the impaction of these teeth is trauma, another reason for the impaction of the left mandibular first premolar was complication of the miniplate fixation. Because one of the miniplate's screws had been inserted under the mandibular first premolar's cervical line, these teeth could not erupt normally. And although the left permanent third molar bud was in the correct position, the right mandibular third molar bud was absent and an odontoma-like malformation had appeared in this area. In addition, the right second molar's distal root had not completely formed. As a result, this patient presents a rare case of the simultaneous occurrence of an odontoma-like malformation and complete and partial arrested root formation, probably as a result of the maxillofacial trauma mentioned in this article. Almost all pediatric fractures must be managed with closed reduction as much as possible. However, if it is necessary to perform an open reduction, careful attention must be paid when the osteosynthetic plates and screws are placed; and the tooth buds' development must be followed periodically.

\section{References}

1. Nelson-Filho P, Silva RA, Faria G, Freitas AC. Odontoma-like malformation in a permanent maxillary central incisor subsequent to trauma to the incisor predecessor. Dent Traumatol. 2005;21:309-12.

2. Suei Y, Mallick PC, Nagasaki T, Taguchi A, Fujita M, Tanimoto K. Radiographic evaluation of the fate of developing tooth buds on the fracture line of mandibular fractures. J Oral Maxillofac Surg. 2006;64:94-9.

3. Tozoglu S, Yolcu U, Tozoglu U. Developmental disturbance of maxillary lateral incisor after trauma. Dent Traumatol. 2007;23:85-6.

4. Ranta R, Ylipaavalniemi P. The effect of jaw fractures in children on the development of permanent teeth and the occlusion. Proc Finn Dent Soc. 1973;69:99-104.

5. Koenig WR, Olsson AB, Pensler JM. The fate of developing teeth in facial trauma: tooth buds in the line of mandibular fractures in children. Ann Plast Surg. 1994;32:503-5.

6. Nixon F, Lowey MN. Failed eruption of the permanent canine following open reduction of a mandibular fracture in a child. Br Dent J. 1990;168:204-5. 